\title{
Generic dependence of the frequency-size distribution of earthquakes on depth and its relation to the strength profile of the crust
}

\author{
M. Spada, ${ }^{1,2}$ T. Tormann, ${ }^{1}$ S. Wiemer, ${ }^{1}$ and B. Enescu ${ }^{3,4}$ \\ Received 11 October 2012; revised 10 December 2012; accepted 12 December 2012; published 28 February 2013.
}

[1] We explore the idea that the relative size distribution of earthquakes, quantified using the so-called $b$-value, is negatively correlated with differential stress. Because the maximum possible differential stress increases linearly in the brittle upper crust, we expect to find a decrease of $b$ with depth. We test this expectation for seven continental areas around the world, each of which is described by a regional earthquake catalog. We find a monotonic decrease in $b$-value between 5 and $15 \mathrm{~km}$ depth. The decrease stops near the brittle-ductile transition. We specifically focus on the high-quality catalogs of earthquakes in California to perform a sensitivity test with respect to depth uncertainty; we also estimate the probability-depth gradient for the occurrence of a target magnitude event and study the behavior of $b$ with depth in near- and off-fault zones. We also translate the observed $b$-depth gradients into $b$-differential stress gradients. Our findings suggest that $b$-values are negatively correlated with differential stress and hus have the potential to act as stress meters in the Earth's crust. Citation: Spada, M., T. Tormann, S. Wiemer, and B. Enescu (2013), Generic dependence of the frequency-size distribution of earthquakes on depth and its relation to the strength profile of the crust, Geophys. Res. Lett., 40, 709-714, doi:10.1029/ 2012GL054198.

\section{Introduction}

[2] Several studies have suggested a relation between differential stress $(\Delta \sigma)$ and the relative size distribution of earthquakes quantified by the $b$-value (e.g., Tormann et al. [2012] and references therein). The $b$-value is the slope of a frequency-magnitude distribution (FMD) when plotted in log-linear space and describes the relative frequency of small- versus large-magnitude earthquakes (Gutenberg and Richter [1944]): $\log N=a-b M$, where $N$ is the number of events greater or equal magnitude $M$ and $a$ describes the total number of earthquakes.

[3] Studies of $b$ range in scale from laboratory rock specimens (e.g., Scholz [1968]) to observations in a variety of

\footnotetext{
All Supporting Information may be found in the online version of this article. ${ }^{1}$ Swiss Seismological Service, ETH Zurich, Zurich, Switzerland.

${ }^{2}$ Now at the Laboratory for Energy Systems Analysis, Paul Scherrer Institute, Villigen PSI, Switzerland.

${ }^{3}$ Faculty of Life and Environmental Sciences, University of Tsukuba, Tsukuba, Japan.

${ }^{4}$ National Research Institute for Earth Science and Disaster Prevention (NIED), Tsukuba, Japan.
}

Corresponding author: Laboratory for Energy Systems Analysis, Paul Scherrer Institute, OHSA D19, CH-5232, Villigen PSI, Switzerland. (Matteo.Spada@psi.ch)

(C)2013. American Geophysical Union. All Rights Reserved. 0094-8276/13/2012GL054198 different tectonic regimes (e.g., Wiemer and Wyss [2002]). In the laboratory, acoustic emissions (AEs) from seismically active micro-cracks during different stages of fracture experiments follow a power law analogous to the Gutenberg-Richter (GR) relation with decreasing $b$-value for increasing $\Delta \sigma$ (e.g., Scholz [1968], Amitrano [2003]).

[4] For induced and natural seismicity, the same relationship was suggested by numerous studies. The $b$-values of earthquakes induced by high-pressure injections in geothermal reservoirs have been documented to be high compared to the regional level (Bachmann et al. [2012]). Asperities - areas on a fault that are strong and therefore slip resistant-have been mapped with low $b$, indicating that they are highly stressed (e.g., Oncel and Wyss [2000], Tormann et al. [2012]). More evidence for this relationship was drawn from seismicity in different tectonic regimes, which according to the brittle failure criterion require different levels of $\Delta \sigma$ (e.g., Scholz [2002]). Natural seismicity in California, Japan, Italy, and worldwide has been documented to exhibit increasing $b$ moving from thrust to strike-slip to normal regimes (Schorlemmer et al. [2005], Gulia and Wiemer [2010], Yang et al. [2012]), again consistent with an inverse $b-\Delta \sigma$ relation.

[5] A generic and rather well-modeled gradient of $\Delta \sigma$ is the strength profile of the lithosphere. Because in equilibrium $\Delta \sigma$ cannot exceed the strength of rocks, profiles of maximum possible $\Delta \sigma$ can be estimated, which increase inearly through the brittle upper crust down to about $15 \mathrm{~km}$ and start decreasing nonlinearly at the brittle-ductile transition (Figure 1a) (e.g., Scholz [2002]).

[6] The signal of increasing $\Delta \sigma$ with depth should be resolvable in the $b$-value distribution with depth, if the latter is indeed sensitive to the former. Some studies have addressed the depth dependence of $b$ in California: Mori and Abercrombie [1997] found a systematic decrease of $b$ with depth, which they explained by a possible decrease of heterogeneity with depth. Gerstenberger et al. [2001] studied the $b$-value variation with depth in California by mapping the ratio between the $b$ estimated for a shallow layer (0-5 km depth) and the one estimated for a deep layer $(7-15 \mathrm{~km})$. Their maps show a strong local variation of the $b$-ratio, which the authors related to the differences in stress level, and about $32 \%$ of the area showed a decrease of the $b$-value with depth. Amorese et al. [2010] studied the variation of $b$-value with depth in seven selected areas in southern California, which according to Gerstenberger et al. [2001] showed $b$-ratio values higher than 1, i.e., a decrease of $b$ with depth. In contrast to the previous studies, Amorese et al. [2010] did not find a statistically significant decrease of $b$ with depth.

[7] This study presents $b$-depth gradients from seven continental areas around the world, each described by 

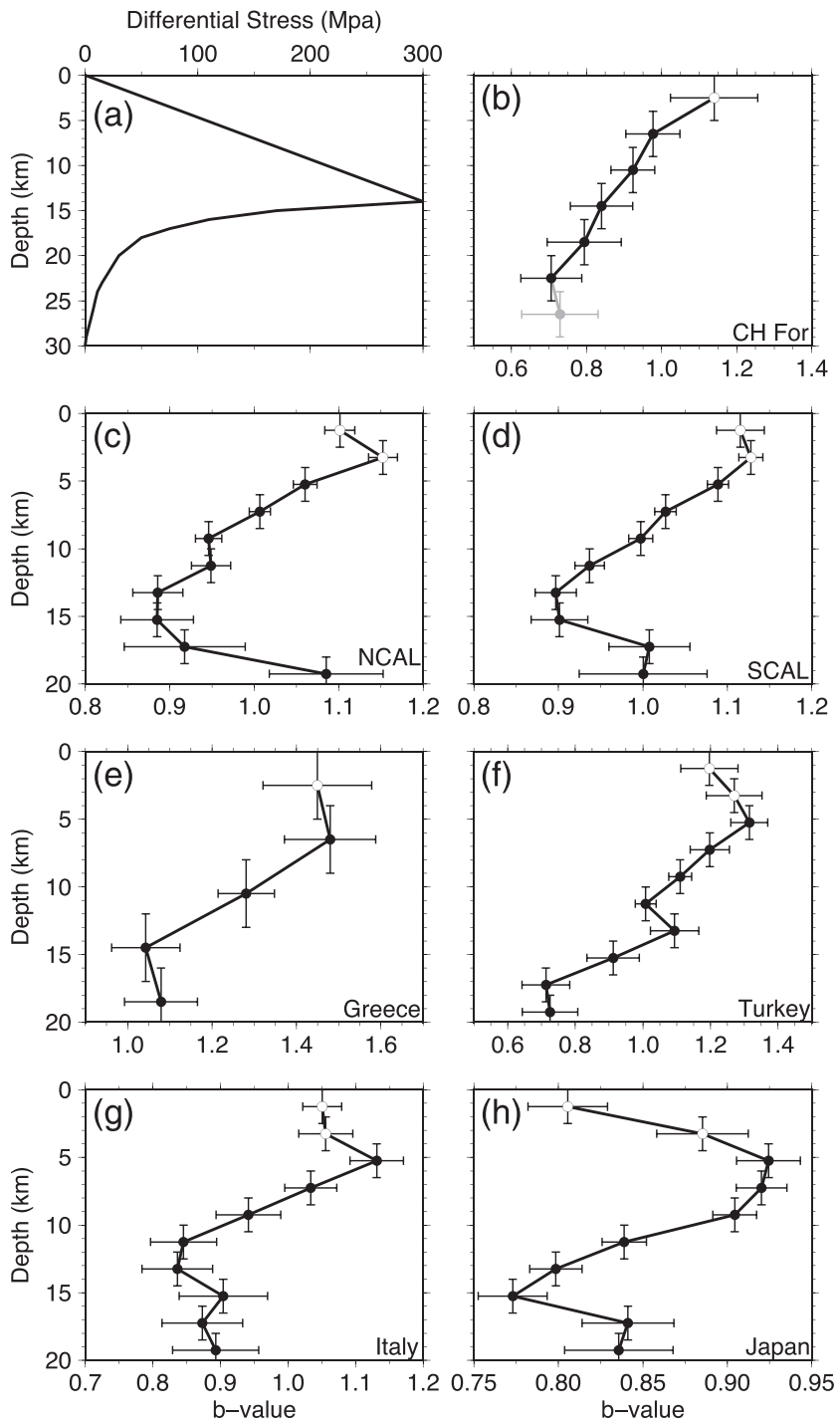

Figure 1. (a) Schematic and simplified illustration of the strength profile of the crust modified after Scholz [2002]. (b-h) The $b$-depth gradients observed for different catalogs. Open circle data points are not taken into account in this study since in those depth layers seismicity is sparse and likely influenced by, for example, geothermal related activity (see text). Vertical error bars: depth layer in which $b$ is estimated. Horizontal error bars: formal uncertainty in $b$ estimates following Shi and Bolt [1982]. (b) Switzerland, restricted to foreland $\left(5.91^{\circ} \mathrm{W}-46.18^{\circ} \mathrm{N} ; 6.145^{\circ} \mathrm{E}-46.886^{\circ} \mathrm{N}\right.$; $7.08^{\circ} \mathrm{E}-47.61^{\circ} \mathrm{N} ; 8.58^{\circ} \mathrm{E}-47.86^{\circ} \mathrm{N} ; 9.86^{\circ} \mathrm{E}-47.62^{\circ} \mathrm{N} ; 6.08^{\circ}$ $\left.\mathrm{E}-46.08^{\circ} \mathrm{N}\right), b$ is computed using events above $M_{c}=2.0$, black: $N_{\min }=50$ events, grey: $N_{\min }=30$ events. (c) Northern California, $M_{c}=2.5$. (d) Southern California, $M_{c}=2.5$. (e) Greece, $M_{c}=4.5$. (f) Turkey, $M_{c}=3.7$. (g) Italy, $M_{c}=2.5$, restricted to mainland. (h) Japan, $M_{c}=2.5$.

regional earthquake catalogs. We apply a bulk analysis to each of the considered regions to demonstrate a generic property of the crust. We do not use a mapping approach since lateral variations of $b$ may in their amplitude dominate over a generic depth gradient, as demonstrated by the spatial variation reported by Gerstenberger et al. [2001] and Amorese et al. [2010]. We specifically focus on the high- quality California catalogs to understand the differences of $b$-depth gradients for near- and off-fault seismicity. Fault zones are special structures in the crust which might be controlled by different processes than off-fault areas, and for southern California fault zones have been shown to have generally lower $b$-values (Page et al. [2011]). We conclude this paper by translating the observed $b$-depth gradient into a $b-\Delta \sigma$ gradient.

\section{Earthquake Catalogs}

[8] We consider earthquake catalogs from different regions around the world. To ensure highest quality locations and completeness levels, we filter all catalogs to contain only onshore events within national boundaries and in the shallow part of the crust, i.e., maximum $20 \mathrm{~km}$ depth. In particular, we use catalogs from the seven regions described below.

[9] Northern and southern California: we use the doubledifference relocated northern Californian catalog (NCAL) from Waldhauser and Schaff [2008], covering 1984-2009, and the waveform relocated southern Californian catalog (SCAL) from 1981 to 2011 (Hauksson et al. [2012]) both filtered to their respective authoritative regions (http:// www.ncedc.org/anss/anss-detail.html). Based on the 3D description of California's major fault structures (243 segments documented in the UCERF3 model), we separate the NCAL and SCAL into near-fault and off-fault seismicity in order to avoid possible mixing between near- and off-fault seismicity. To avoid possible mixing between near- and off-fault events within the seismic damage zone (Hauksson [2010]), we regard all events within $2 \mathrm{~km}$ of the fault plane as near-fault seismicity, as suggested by Powers and Jordan [2010], and following Hauksson [2010] we select events further than $10 \mathrm{~km}$ from the fault plane as off-fault seismicity.

[10] Switzerland: we use the instrumental seismicity record (1975-2009) of the ECOS09 earthquake catalog (Fäh et al. [2011]) restricted to the subset of the foreland region (CH For) (Figure 1).

[11] Italy: we use the relocated Italian Seismicity Catalogue (C.S.I.) version 1.1 (available on http://csi.rm.ingv.it) from 1981 to 2002 considering only events on the mainland.

[12] Greece: we use the Hellenic Arc area of the catalog by Papazachos et al. [2009] from 1975 to 2009.

[13] Turkey: we use the earthquake catalog compiled by the Kandilli Observatory and Earthquake Research Institute Department of Earthquake Engineering (KOERI) from 1990 to 2011 (available on http://www.koeri.boun.edu.tr/ sismo/indexeng.htm).

[14] Japan: we use the Japan Meteorological Agency (JMA) catalog from 1997 to 2011.

\section{Method}

[15] The correct estimate of the $b$-value depends critically on the assessment of a catalog's magnitude of completeness $\left(M_{c}\right)$ (Wiemer and Wyss [2002]): if $M_{c}$ is underestimated, $b$ will be biased toward lower values. $M_{c}$ varies as a function of space and time throughout all earthquake catalogs. In this study, we estimate conservative completeness levels, i.e., we analyze $M_{c}$ variation through time using the maximum curvature method with a correction factor of 0.2 (e.g., Wössner and Wiemer [2005]), and choose the maximum estimates of 
each time series. Furthermore, we verify that this estimate is also greater than the maximum $M_{c}$ observed with depth.

[16] We estimate $b$-values for sample sizes greater than 50 events $\left(N_{\min }=50\right)$ above $M_{c}$, using the maximum likelihood method (Aki [1965]) and compute the formal uncertainty in $b$ using the equation proposed by Shi and Bolt [1982]. This error estimate is mainly reflecting the total number of events.

[17] In this study, $b$-values are estimated for a given number of overlapping depth layers. We choose a layer width of $2.5 \mathrm{~km}$ and an overlap value of one fifth of the width. The influence of the choice of the layer width on the results is shown in Figures $3 a$ and $3 b$ for NCAL and SCAL.

[18] We also check the sensitivity of our analyses with respect to depth location uncertainty. For each depth location in the NCAL and SCAL catalogs, we simulate 1000 depth values from a Gaussian distribution with the catalog value treated as the mean and with various values of the standard deviation $(0.5,1,2,3,4$, and $5 \mathrm{~km})$. From the resulting simulated catalogs, we estimate the mean and the standard deviation for the $b$-value with depth related to each uncertainty value (Figures $3 \mathrm{c}$ and $3 \mathrm{~d}$ ).

\section{A Generic Depth Gradient}

[19] For all considered earthquake catalogs, we observe similar depth gradients showing a generic decay of $b$ with increasing depth from 5 to $15 \mathrm{~km}$ (Figures $1 \mathrm{~b}-1 \mathrm{~h}$ ). The variation of the $b$-depth gradients between regions is strongly influenced by the magnitude scale used for the local earthquake catalogs as well as by the main tectonic regime acting in the study area. The $b$-value results are well constrained in this $5-15 \mathrm{~km}$ depth range (Figure 2 for NCAL and SCAL), uncertainties are small and, for NCAL and SCAL, $90 \%$ of the seismicity is shallower than $\sim 17 \mathrm{~km}$ (Figures 3e and 3f). Outside the depth range 5-15 km, the fits of FMDs above $M_{c}$ do not always obey a power law, e.g., we note that the uppermost data points (above $5 \mathrm{~km}$ ) in Figure 1 (open circles) have to be considered with caution. Such shallow seismicity is sparse and likely influenced by, for example, geothermal related activity, as we have verified

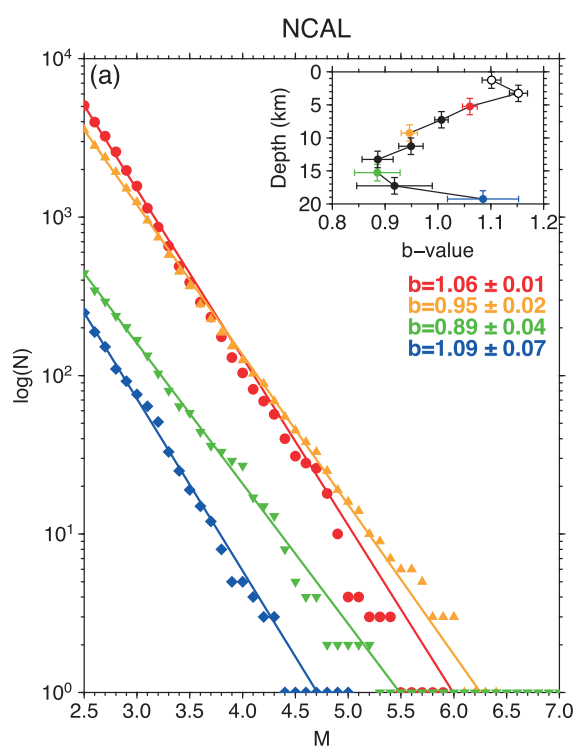

in the cases of NCAL and SCAL. We show (Figures 3c and $3 \mathrm{~d}$ ) that the effect of location error in depth is minor: the generic $b$-depth gradient is still present considering different location errors.

[20] Knowing the strength gradient of the crust, we interpret the turning point of the $b$-values at a depth of $\sim 15 \mathrm{~km}$ as the transition from brittle behavior of the upper crust to ductile behavior below. The $b$-depth gradient therefore allows an estimate of this transition depth, i.e., an estimate of the thickness of the brittle crust in different regions of the world. As has been suggested before (Deichmann [1992]), this brittle-ductile transition is located significantly deeper in the Swiss foreland. Here, we cannot resolve the transition depth with our $b$-values if we require $N_{\min }=50$ events in each depth layer, which we normally do to keep uncertainties reasonably low. An experiment of reducing $N_{\min }$ for this data set to 30 events allows us to indeed resolve an additional $b$-value for a deeper layer and the anticipated - if only slight - increase, which suggests a depth of the brittle-ductile transition of about $25 \mathrm{~km}$ for the foreland in Switzerland (Figure 1b).

[21] Combining the $b$-depth gradient with the activity rate ( $a$-value), we estimate the probability-depth gradient for the occurrence of one or more events of a target magnitude $\left(M_{\text {targ }}\right)$. In more detail, for each depth layer, we first calculate from the annual activity rates combined with the $b$-value the expected recurrence time for $M_{\text {targ. }}$. We then use the determined recurrence time to calculate the occurrence probability of one more event with magnitude $M_{\text {targ }}$ per year (Figures $3 \mathrm{~g}$ and $3 \mathrm{~h}$ ). The shape of the probability-gradient is quite different in the two test cases. While for SCAL the maximum probabilities of having large, $M \geq 6$, and small, $M \leq 4$, events are well distinguished in depth $(\sim 10$ and $\sim 7 \mathrm{~km}$ depth, respectively), there is no remarkable separation for NCAL. In addition, these results are still different from the usually assumed constant $b=1$ for all depths. The $b=1$ curve shows a higher probability shallower with respect to the other cases (Figures $3 \mathrm{~g}$ and $3 \mathrm{~h}$ ).

[22] Separating near-fault from off-fault seismicity in California, we see similar gradients in both settings (Figures $3 \mathrm{i}$

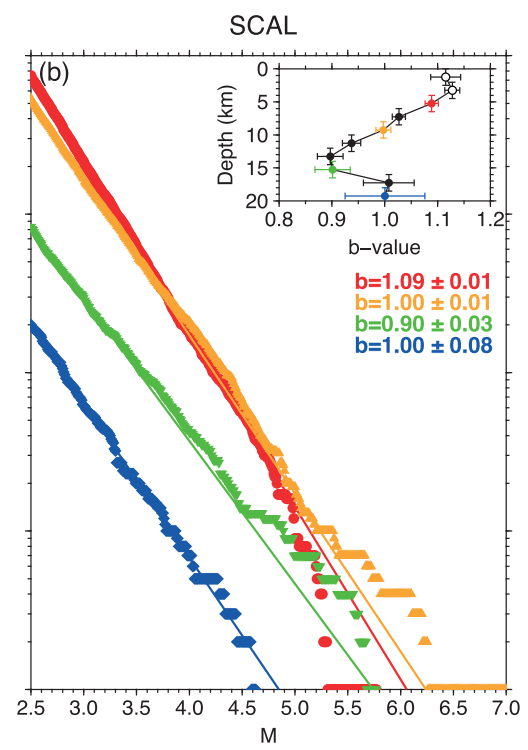

Figure 2. Cumulative FMDs above $M_{c}$ for (a) NCAL and (b) SCAL for four depth layers together with the estimated $b$-values. Inset: $b$-depth gradients as shown in Figure 1; in color, the depth layers corresponding to the FMDs. 

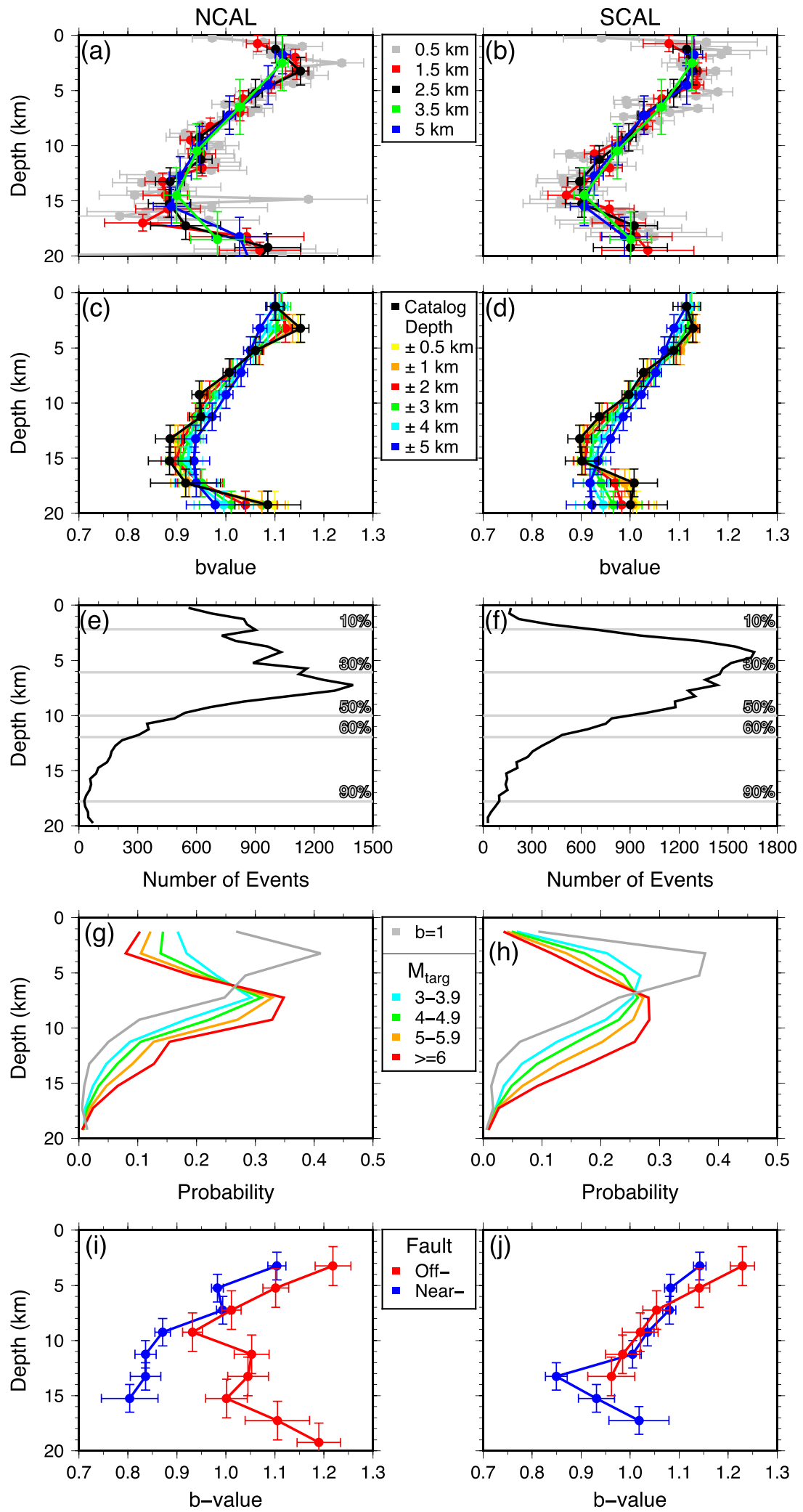

Figure 3. NCAL (left column) and SCAL (right column). (a and b) $b$-depth gradients for different choices of layer width. (c and d) $b$-depth gradients for different levels of assumed depth location uncertainty. (e and f) Numbers of observed events at depth (in steps of $0.5 \mathrm{~km}$ ). Grey lines: percentiles for the numbers of observed events. ( $\mathrm{g}$ and $\mathrm{h}$ ) Probability-depth gradient for the occurrence of one or more events of magnitude $M_{\text {targ }}$, derived from the $a$ - and $b$-values estimated in each depth layer. (i and j) $b$-depth gradients for near-fault (blue) and off-fault seismicity (red). 
and $3 \mathrm{j}$ ). In southern California (Figure 3i), they appear to be undistinguishable, although the bulk $b$-values using all depths reproduce the differences documented by Page et al. [2011], that is, the near-fault $b$-value is 1.04 , while the off-fault value is 1.13 . The difference in the bulk values is similar in northern California, 0.98 and 1.06, near-fault and off-fault, respectively. However, the gradients here suggest consistently lower $b$-values at all depths along faults compared to off-fault seismicity, which agrees with the suggestion that larger magnitude events are more likely to occur on major faults than off-fault.

[23] Assuming hydrostatic pressures and Byerlee friction coefficient $(\mu=0.75)$, the $\Delta \sigma$ gradient for a strike-slip regime is approximately $-20 \mathrm{MPa} / \mathrm{km}$, which agrees with in situ measurements of stress gradients in several boreholes with maximum depths of $\sim 8 \mathrm{~km}$ (e.g., Zoback and Townend [2001]). Merging this $\Delta \sigma$-depth gradient and the resolved $b$-depth gradients (Figure 1) by assigning to a $b$-value at a given depth the corresponding $\Delta \sigma$ at the same depth, we can translate the $b$-depth into a $b-\Delta \sigma$ gradient. In this setting, the amplitude of $b$ is unimportant, so we normalize all $b$-values to the first point below $5 \mathrm{~km}$ depth and consider these normalized $b-\Delta \sigma$ gradients for depths from 5 to $15 \mathrm{~km}$, which suggest a possibly linear inverse relationship $b-\Delta \sigma$ (Figure 4). These results are in agreement with the previously suggested negative correlation $b-\Delta \sigma$ for natural earthquakes (e.g., Schorlemmer et al. [2005]). This generic $b-\Delta \sigma$ relationship for natural earthquakes is thus consistent with findings from laboratory experiments (Amitrano [2003]).

[24] Even if we find a generic decrease of $b$ with $\Delta \sigma$ for all considered earthquake catalogs, we only consider the

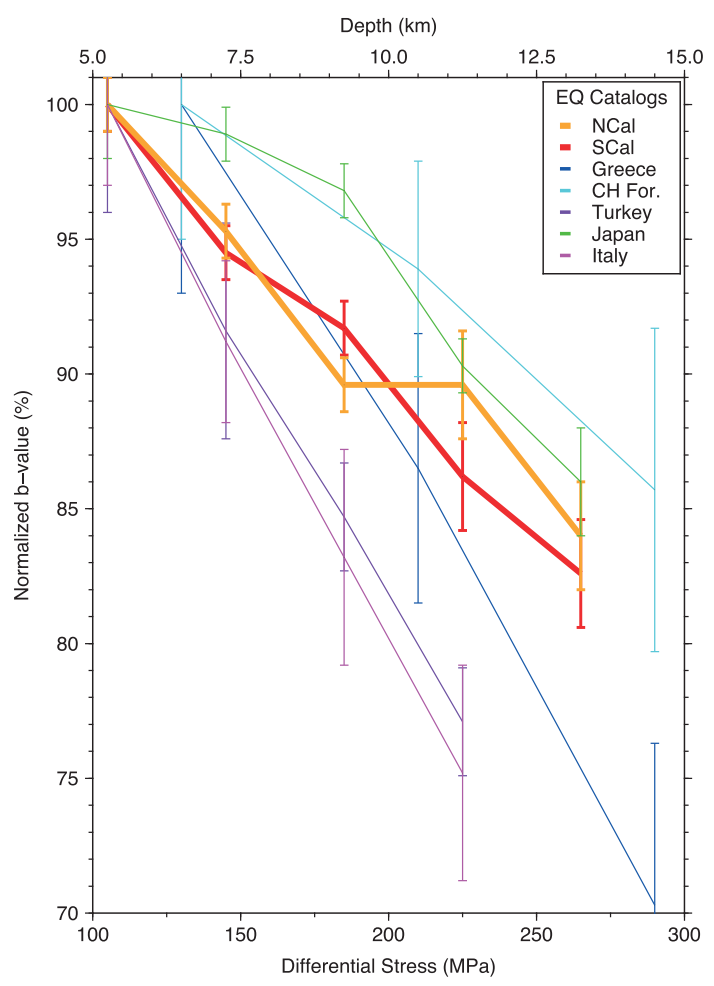

Figure 4. Normalized $b$-value to the first point (deeper than $5 \mathrm{~km}$ depth) in percentage versus $\Delta \sigma$ for the seven considered earthquake catalogs. Bold lines correspond to the high-quality relocated earthquake catalogs for northern and southern California. high-quality relocated earthquake catalogs of northern and southern California to try to quantify the possibly linear relationship between the two parameters. Stacking the two gradients, the general slope, fit by a least squares method, suggests that $b$ might be negatively correlated to $\Delta \sigma$ as $-10^{-3}$ $\mathrm{MPa}$. We note, though, that this is a damped value: this relation could be much flatter if more localized data would be used, as this could resolve a stronger local $b$-value variation.

\section{Conclusions}

[25] The general inverse relation $b-\Delta \sigma$ suggested by laboratory experiments holds true for tectonic earthquakes. By applying a bulk analysis of regional catalogs we eliminate local variations; we do this because Gerstenberger et al. [2001] and Amorese et al. [2010] showed that lateral variations of $b$ may in their amplitude dominate over a generic depth gradient. This bulk analysis also reveals a generic decrease of $b$ with depth through the brittle part of the crust.

[26] For all studied regions except the Swiss foreland, we find a turning point in the gradient at depths of $\sim 15 \mathrm{~km}$, which we interpret as the brittle-ductile transition. The $b$-value derived transition depth for the Swiss foreland is deeper and with $\sim 25 \mathrm{~km}$ depth consistent with former suggestions strengthening the robustness of our interpretation. Our observation thus confirms the potential of $b$-values to act as a stress meter in the Earth's crust.

[27] Acknowledgments. The presented work was supported by the Swiss Federal Nuclear Safety Inspectorate (ENSI) and the Swiss National Foundation (SNF) grant PMPDP2 134174. We would like to thank A. Mignan, T. H. W. Göbel and J. Zechar for helping to improve the manuscript and J. Wössner for useful discussions. We thank J. Townend and an anonymous reviewer for their helpful comments. We thank the Japan Meteorological Agency (JMA) for providing the earthquake catalog used in this study.

\section{References}

Aki, K. (1965), Maximum likelihood estimate of $\mathrm{b}$ in the formula $\log \mathrm{N}=a-\mathrm{bM}$ and its confidence limits, Bull, Earthq, Res, Inst., 43, 237-239.

Amitrano, D. (2003), Brittle-ductile transition and associated seismicity: Experimental and numerical studies and relationship with the $b$ value, J. Geophys. Res., 108(B1), 2044, doi:10.1029/2001JB000680.

Amorese, D., J. R. Grasso, and P. A. Rydelek (2010), On varying b-values with depth: results from computer-intensive tests for southern California, Geophys, J, Int., 180(1), 347-360, doi:10.1111/j.1365-246X.2009.04414.x.

Bachmann, C. E., S. Wiemer, B. P. Goertz-Allmann, and J. Woessner (2012), Influence of pore-pressure on the event-size distribution of induced earthquakes, Geophys. Res. Lett., 39, L09302, doi:10.1029/ 2012GL051480.

Deichmann, N. (1992), Structural and rheological implications of lowercrustal earthquakes below northern Switzerland, Phys. Earth Planet. Inter., 69 (3-4), 270-280, doi:10.1016/0031-9201(92)90146-M.

Fäh, D., D. Giardini, P. Kästli, N. Deichmann, M. Gisler, G. Schwarz-Zanetti, S. Alvarez-Rubio, S. Sellami, B. Edwards, B. Allmann, F. Bethmann, J. Wössner, G. Gassner-Stamm, S. Fritsche, and D. Eberhard (2011), ECOS-09 Earthquake Catalogue of Switzerland Release 2011. Report and Database, Tech. Rep.

Gerstenberger, M., S. Wiemer, and D. Giardini (2001), A systematic test of the hypothesis that the b value varies with depth in California, Geophys. Res. Lett., 28(1), 57-60.

Gulia, L. and S. Wiemer (2010), The influence of tectonic regimes on the earthquake size distribution: A case study for Italy, Geophys. Res. Lett., 37, L10305, doi:10.1029/2010GL043066.

Gutenberg, R. and C. Richter (1944), Frequency of earthquakes in California, Bull. Seismol. Soc. Am., 34, 185-188.

Hauksson, E. (2010), Spatial separation of large earthquakes, aftershocks, and background seismicity: Analysis of interseismic and coseismic 


\section{SPADA ET AL.: THE $B$-VALUES WITH DEPTH}

seismicity patterns in southern California, Pure Appl. Geophys., 167, 979-997, doi:10.1007/s00024-010-0083-3.

Hauksson, E., W. Yang, and P. M. Shearer (2012), Waveform relocated earthquake catalog for Southern California (1981 to June 2011), Bull. Seismol. Soc. Am., 102(5), 2239-2244.

Mori, J. and R. Abercrombie (1997), Depth dependence of earthquake frequency-magnitude distributions in California: Implications for rupture initiation, J. Geophys. Res., 102(B7), 15081-15090.

Oncel, A. O. and M. Wyss (2000), The major asperities of the $1999 \mathrm{Mw}=7.4$ Izmit earthquake defined by the microseismicity of the two decades before it, Geophys. J. Int., 143(3), 501-506.

Page, M. T., D. Alderson, and J. Doyle (2011), The magnitude distribution of earthquakes near southern California faults, J. Geophys. Res. 116 , B12309, doi:10.1029/2010JB007933.

Papazachos, B. C., P. E. Comninakis, E. M. Scordilis, G. F. Karakaisis, and C. B. Papazachos (2009), A catalogue of earthquakes in the Mediterranean and surrounding area for the period 1901-Sep 2009, Publ. Geoph. Lab., University of Thessaloniki.

Powers, P. M. and T. H. Jordan (2010), Distribution of seismicity across strike-slip faults in California, J. Geophys. Res., 115, B05305, doi:10.1029/2008JB006234.

Scholz, C. (1968), The frequency-magnitude relation of microfracturing in rock and its relation to earthquakes, Bull. Seismol. Soc. Am., 58(1), 399-415.

Scholz, C. (2002), The Mechanics of Earthquakes and Faulting, 2nd ed. Cambridge University Press, New York.
Schorlemmer, D., S. Wiemer, and M. Wyss (2005), Variations in earthquake-size distribution across different stress regimes, Nature, 437 (7058), 539-542, doi:10.1038/nature04094.

Shi, Y. and B. Bolt (1982), The standard error of the magnitude-frequency $b$ value, Bull. Seismol. Soc. Am., 72(5), 1677-1687.

Tormann, T., S. Wiemer, and J. Hardebeck (2012), Earthquake recurrence models fail when earthquakes fail to reset the stress field, Geophys. Res. Lett., 39, L18310, doi:10.1029/2012GL052913.

Waldhauser, F. and D. P. Schaff (2008), Large-scale relocation of two decades of northern California seismicity using cross-correlation and double-difference methods, J. Geophys. Res., 113, B08311, doi:10.1029/ 2007JB005479.

Wiemer, S. and M. Wyss (2002), Mapping spatial variability of the frequency-magnitude distribution of earthquakes, Adv. Geophys., 45, 259-302, doi:10.1016/S0065-2687(02)80007-3.

Wössner, J. and S. Wiemer (2005), Assessing the quality of earthquake catalogues: Estimating the magnitude of completeness and its uncertainty, Bull. Seismol. Soc. Am., 95(2), 684-698, doi: 10.1785/ 0120040007.

Yang, W., E. Hauksson, and P. M. Shearer (2012), Computing a large refined catalog of focal mechanisms for southern California (1981-2010): Temporal stability of the style of faulting, Bull. Seismol. Soc. Am., 102(3), doi: $10.1785 / 0120110311$.

Zoback, M. D. and J. Townend (2001), Implications of hydrostatic pore pressures and high crustal strength for the deformation of intraplate lithosphere, Tectonophysics, 19-30. 